\title{
Stabilization of a dynamically unstable opto-thermo-mechanical oscillator
}

\author{
Wenle Weng,,${ }^{1,2, *}$ James D. Anstie, $^{1,2}$ Paul Abbott, ${ }^{2}$ B. Fan, ${ }^{3,4}$ T. M. Stace, ${ }^{4}$ and Andre N. Luiten ${ }^{1,2}$ \\ ${ }^{1}$ Institute for Photonics and Advanced Sensing and School of Chemistry and Physics, University of Adelaide, South Australia 5005, Australia \\ ${ }^{2}$ School of Physics, University of Western Australia, Western Australia 6009, Australia \\ ${ }^{3}$ College of Physics, Communication and Electronics, Jiangxi Normal University, Nanchang 330022, China \\ ${ }^{4}$ ARC Centre for Engineered Quantum Systems, University of Queensland, Brisbane 4072, Australia
}

(Received 19 December 2014; published 1 June 2015)

\begin{abstract}
We theoretically and experimentally examine thermal oscillations in a calcium fluoride whispering-gallerymode resonator that lead to strong mode-frequency oscillations. We show that these oscillations arise from interplay among thermal expansion, the thermo-optic effect, and Kerr effects. In certain regimes we observe chaotic behavior and demonstrate that the threshold for this behavior can be predicted theoretically. We then demonstrate a self-stabilization technique that suppresses the oscillations and delivers high temperature and frequency stability without reference to external standards.
\end{abstract}

DOI: 10.1103/PhysRevA.91.063801

PACS number(s): 42.60.Da, 05.45.-a, 42.62.Fi

\section{INTRODUCTION}

Over the last 2 decades there has been strong interest in whispering-gallery-mode (WGM) resonators for both fundamental [1-4] and applied research [5-8]. This interest has been principally motivated because of the small size of the resonator together with their high quality factor $(Q)$. This combination gives rise to large circulating powers and strong nonlinear effects at modest input powers.

Calcium fluoride $\left(\mathrm{CaF}_{2}\right)$ has a number of attributes that make it almost ideal as a resonator material: its ultralow intrinsic absorption and ability to take a high polish lead to extremely low overall optical dissipation [9]. $\mathrm{CaF}_{2}$ resonators have thus found broad applications, in frequency stabilization [10], frequency comb generation [11], stimulated Raman scattering [12], surface acoustic wave generation [13], and cavity optomechanics [14]. Unfortunately, $\mathrm{CaF}_{2}$ also has an unusual characteristic in that the temperature dependence of its refractive index (thermo-optic effect) and its linear dimensions (thermal expansion) have opposite signs. This characteristic has generally limited high-power applications for $\mathrm{CaF}_{2}$ as it leads to pronounced nonlinear dynamical instabilities. In this article we report on observations of these instabilities and demonstrate a technique for stabilizing the dynamics.

Self-generated thermal oscillations have previously been observed in heterogenous resonators [15] as well as in resonators with complex material properties [16-18]. We generalize those observations by observing these mode-frequency oscillations when the probing laser frequency is scanned across the resonance at various tuning rates. Then we develop a model that shows that the oscillations arise from competition among the thermal expansion, the thermo-optic effect, and Kerr effects - furthermore, we can obtain the magnitude and response times of these effects from our observations. The model can also predict the complex self-sustained oscillations and self-pulsation in silicon microresonators that have been observed by others $[19,20]$. We furthermore observe that under some conditions the thermal oscillations enter into a chaotic regime: we show that our model equations predict

\footnotetext{
*wenle.weng@adelaide.edu.au
}

the threshold pumping conditions for the limit cycles together with their natural frequency. Finally, we take the advantage of the wavelength dependence of the thermo-optic coefficients to develop a dual-mode self-thermal-locking technique. We show that this technique enables high-level laser power to be coupled into the resonator without the strong oscillations that would normally occur. In addition, this approach stabilizes both the temperature and the resonance frequency of the resonator without the need for any auxiliary temperature or frequency measurements.

\section{SELF-OSCILLATION DYNAMICS}

Figure 1 shows the experimental setup for studying the oscillatory behavior of $\mathrm{a} \mathrm{CaF}_{2} \mathrm{WGM}$ resonator. A high-index prism was used to couple the output of a Nd:YAG laser into a mode. The gap between the prism and the resonator was adjusted by a piezoelectric positioning stage to set the coupling condition [21]. The resonator was glued to an optical post which was temperature controlled by a thermistor-heater pair. The transmitted light, which arises from interference between the input laser light and the out-coupled light from the resonator, was registered on a photodetector. We initially used a very low incident power and weak coupling to obtain an unperturbed (angular) mode frequency of $\omega_{0}$ along with an unperturbed mode bandwidth of $\delta=\omega_{0} / Q=2 \pi \times 400 \mathrm{kHz}$, i.e., $Q \sim 7 \times 10^{8}$. Figure 2(a) shows a closeup photograph of the resonator. The resonator has a radius of $5 \mathrm{~mm}$ and a thickness of $1 \mathrm{~mm}$. Following the initial shaping with a single-point diamond turning machine, the surface was hand polished with diamond paste with a grit size of $1 \mu \mathrm{m}$. Figure 2(b) shows the transmission spectrum of one mode in the resonator measured at low power.

To characterize the thermal and optical nonlinearities, we increase the laser power and resonator coupling. In some WGM resonators (e.g., $\mathrm{MgF}_{2}$, silica), nonlinearities have been used to spontaneously lock the cavity to a blue-detuned laser, avoiding the need for active stabilization electronics [22,23]. Figure 3(a) shows periodic features that appear when the laser frequency is fixed at a frequency above the heated resonance but $28 \mathrm{MHz}$ below the unperturbed resonance. These self-oscillations indicate that the $\mathrm{CaF}_{2}$ resonator is 


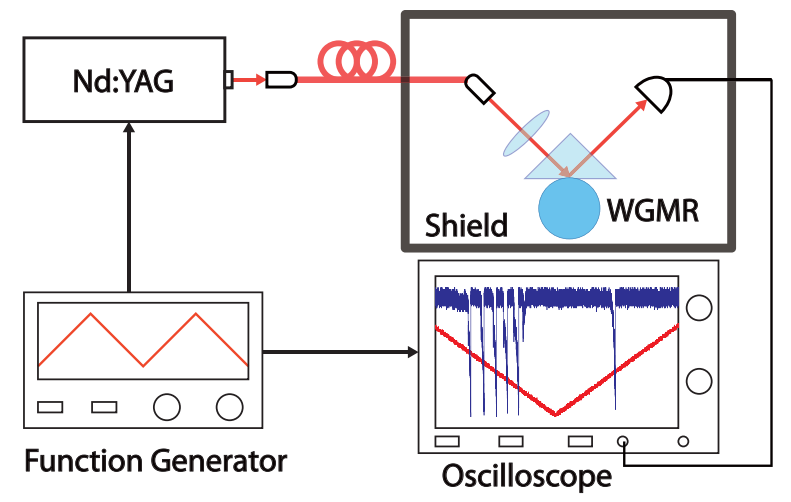

FIG. 1. (Color online) Experimental scheme for studying the thermal oscillations in a $\mathrm{CaF}_{2} \mathrm{WGM}_{\text {resonator. }}$

unstable in this regime and will not spontaneously lock to the laser. There are a variety of other nonlinear phenomena that become evident as we scan the laser across a cavity resonance, shown in the left column of Fig. 3. In what follows, we develop a mathematical model which replicates these experimentally observed phenomena.

Figures 3(c) and 3(e) show quasiperiodic features in the response of the transmission spectra when the laser frequency is slowly scanned downwards across a single mode of the resonator. The shapes of these features arise because the dissipated power results in an increase in the temperature within the mode volume causing a rapid blue-shifting of the resonance frequency through the thermo-optic effect. This is seen as the narrow feature in the leading edge of the resonance feature similar to what has been described as "bandwidth narrowing" [24]. The sharply peaked temperature in the mode volume then diffuses throughout the resonator resulting in a red-shifting of the resonant frequency because the thermal expansion dominates over the thermo-optic effect. This effect leads to the resonant mode "catching up" and passing through the down-scanning laser frequency, causing the broader feature that is seen on the trailing edge of the oscillation feature. This process repeats itself, until heating from the laser can no longer shift the resonant frequency to bring it into resonance with the tuning laser.

Conversely, if the laser frequency is scanned upwards then we see a single resonant feature, shown in Fig. 3(g). The particular shape arises because the initial blue-shifting causes
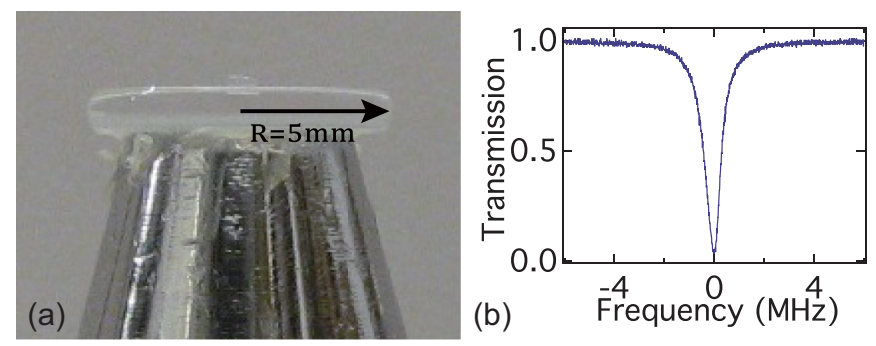

FIG. 2. (Color online) (a) A picture of the resonator. (b) The resonance spectrum of a mode probed by a frequency-sweeping laser with weak intensity.

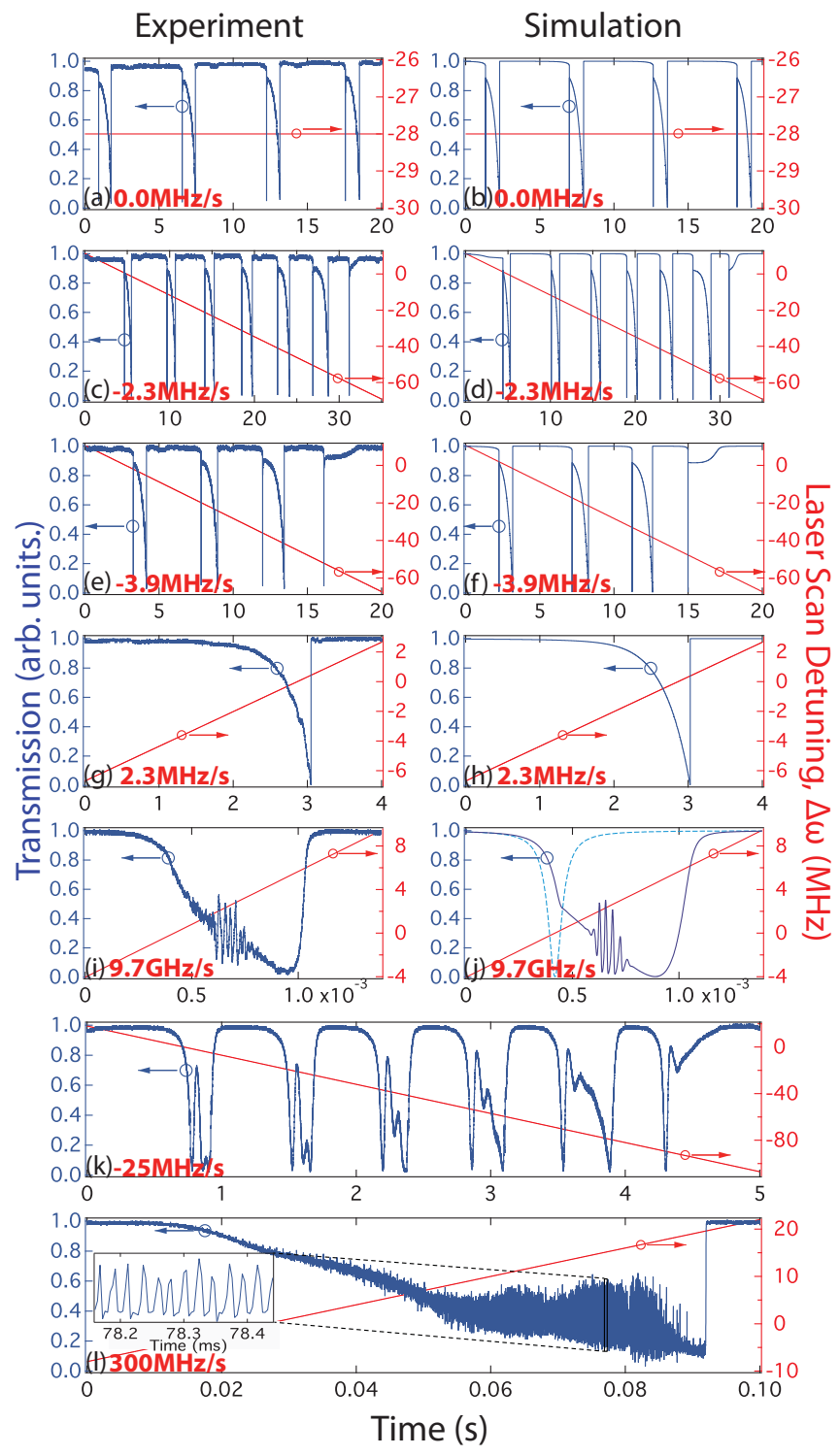

FIG. 3. (Color online) (a) Measured self-oscillation when the laser frequency is fixed at a blue-detuned position from the mode resonance frequency. Panels (c), (e), (g), (i), (k), and (l) are experimental measurements when the laser frequency is scanned at the rates indicated in each panel (text and straight red lines). Panels (b), (d), (f), (h), and (j) are corresponding numerical simulations using Eqs. (1) to (4); the light-blue dashed curve in panel (j) is the mode spectrum with nonlinear effects suppressed, for comparison. The inset of panel (1) is an enlarged fraction of the oscillations. The input power is $50 \mathrm{~mW}$ for panels (a)-(h), $65 \mathrm{~mW}$ for panel (k), and $82 \mathrm{~mW}$ for panels (i) and (1).

the mode to shift with the laser and then shift rapidly in the red direction by thermal expansion.

To explain these observations, we present a model that expands upon earlier approaches [15]. The model includes thermal expansion, the thermo-optic effect, and the Kerr effect. The instantaneous resonant frequency $\omega_{r}(t)$ is

$$
\omega_{r} / \omega_{0}=1-\alpha \Delta T_{r}-\beta \Delta T_{m} / n-n_{\mathrm{k}} P_{c} /(n A),
$$


where $\omega_{0}$ is the cavity resonance frequency (in the absence of nonlinear effects), $n$ is the refractive index, $n_{\mathrm{k}}$ is the Kerr coefficient, $\beta$ is the thermo-optic coefficient, $\alpha$ is the thermal expansion coefficient, $A$ is the effective cross-sectional area of the optical mode, and $\Delta T_{m}$ and $\Delta T_{r}$ are the temperature changes induced by the laser field averaged over the optical mode volume or the whole resonator, respectively. The circulating power is $P_{c}=\left|E_{c}\right|^{2} / \tau_{r}$, where $E_{c}$ is the intracavity field and $\tau_{r}=2 \pi r n / c$ is the round-trip time for a resonator of radius $r$.

The field amplitude, the mode, and the resonator temperatures evolve according to

$$
\begin{gathered}
E_{c}^{\prime}(t)=-[\delta / 2+i \Delta \omega(t)] E_{c}+i \kappa \sqrt{P_{\mathrm{in}}}, \\
\Delta T_{m}^{\prime}(t)=-\gamma_{m}\left(\Delta T_{r}-\Delta T_{m}\right)+\gamma_{\mathrm{abs}} P_{c}, \\
\Delta T_{r}^{\prime}(t)=-\gamma_{r} \Delta T_{r}+\left(V_{m} / V_{r}\right) \gamma_{m}\left(\Delta T_{r}-\Delta T_{m}\right),
\end{gathered}
$$

where $\Delta \omega(t)=\omega_{l}(t)-\omega_{r}(t)$ is the detuning of the laser frequency with respect to the instantaneous mode resonance frequency, $\delta$ is the mode bandwidth, and $P_{\text {in }}=\left|E_{\mathrm{in}}\right|^{2} / \tau_{r}$ is the input power. The coupling coefficient, $\kappa$, that determines the rate of exchange between the mode and the external field is $\kappa=\sqrt{\omega_{r} / Q_{c}}$, where $Q_{c}$ is the coupling $Q$. Heat dissipates from the mode volume to the rest of the resonator bulk at rate $\gamma_{m}$ and from the resonator bulk to the environment at rate $\gamma_{r}$. The conversion of optical energy into heat through absorption is expressed as $\gamma_{\text {abs }}$. We calculate a mode volume $V_{m}=$ $3.7 \times 10^{-11} \mathrm{~m}^{3}$ and a resonator volume $V_{r}=7.85 \times 10^{-8} \mathrm{~m}^{3}$ from a finite element model of the modes and the physical dimensions of the resonator.

We adjust the phenomenological values above to obtain best agreement between the calculations and the corresponding experimental data as shown in Figs. 3(b), 3(d), 3(f), and 3(h), and we find excellent agreement when $\gamma_{m}=3720 / \mathrm{s}, \gamma_{r}=$ $0.102 / \mathrm{s}$, and $\gamma_{\mathrm{abs}}=0.3 \mathrm{~K} / \mathrm{J}$. As a check on the physical realism of these values we obtain independent estimates through a combination of experiment and theory: by observing the response of the resonator frequency following a change in incident power we obtain $\gamma_{r} \approx 0.08 / \mathrm{s}$. We find $\gamma_{m} \approx 10^{3} / \mathrm{s}$ from a finite-element heat-transfer model for the time for heat in the optical mode to dissipate into the bulk resonator. We calculate $\gamma_{\mathrm{abs}}=\alpha_{0} c \tau_{r} /\left(n C_{p} \rho V_{m}\right)$ in terms of the absorption coefficient $\alpha_{0}=2 \times 10^{-5} / \mathrm{cm}$ [25] to obtain $\gamma_{\mathrm{abs}}=0.63 \mathrm{~K} / \mathrm{J}$. These estimates are in reasonable agreement with the values obtained from the best fit in the simulations.

For the conditions in Fig. 3(a) (50 mW input power) the model indicates a $0.028 \mathrm{~K}$ temperature oscillation in the mode volume and a $0.011 \mathrm{~K}$ temperature oscillation in the resonator volume. This combination generates a frequency oscillation of $42 \mathrm{MHz}$. For these slow scan speeds the Kerr effect plays little role in the dynamics and the frequency oscillation principally arises from the interplay of thermal expansion and thermooptic effects.

When the laser frequency is scanned rapidly upwards $(9.7$ $\mathrm{GHz} / \mathrm{s}$ ) across the resonance and with increased input power $P_{\text {in }}(>80 \mathrm{~mW})$, we observe qualitatively different behavior, as shown in Fig. 3(i). At this scan rate, heat dissipation into the environment is negligibly slow, and the interplay between the Kerr effect and the thermo-optical effects produces spontaneous oscillations with frequency $\xi \sim 40 \mathrm{kHz}$ as seen in Fig. 3(j). Our model closely replicates this behavior although we find it necessary to adjust $\gamma_{\mathrm{abs}}=0.045 \mathrm{~K} / \mathrm{J}$, reflecting modifications to the lumped element approach at high frequencies [16,26]. Self-sustained oscillations are also seen at intermediate scan rates, as shown in Figs. 3(k) and 3(1).

The equations for our opto-thermo-mechanical system, Eqs. (1) to (4), are mathematically similar to those that describe the phase-space dynamics of a nonlinear optomechanical oscillator [27] and which have been observed experimentally [28-30]. The dynamics are known to have a Hopf instability, exhibiting limit cycles. We find the critical points in the dynamical system assuming that the resonator temperature deviation is zero, i.e., $\Delta T_{r}=0$. This corresponds to fast dynamics, in which the bulk temperature is essentially constant. We define $T_{1}=\Delta T_{m}$ and rewrite the dynamical equations as

$$
\begin{gathered}
E_{c}^{\prime}(t)=-\{q+2 \pi i[\Delta+f(t)]\} E_{c}(t)+i \kappa \sqrt{P_{\mathrm{in}}}, \\
T_{1}^{\prime}(t)=-\gamma_{m} T_{1}(t)+\gamma_{\mathrm{abs}}\left|E_{c}(t)\right|^{2} / \tau_{r}, \\
f(t)=v_{1} T_{1}(t)+N_{k}\left|E_{c}(t)\right|^{2} / \tau_{r},
\end{gathered}
$$

where $q=q_{0}+q_{c}=\pi f_{r}\left(1 / Q_{0}+1 / Q_{c}\right), v_{1}=f_{r} \beta / n, N_{k}=$ $f_{r} n_{k} /(n A)$, and the detuning is $\Delta=f_{l}-f_{r}$. Note that $\beta<0$, as is $v_{1}$. Numerical values for these parameters are given in Table I.

We find the stationary solutions $\left(T_{\mathrm{S}}, E_{\mathrm{S}}\right)$ by solving Eq. (5) and Eq. (6) with $T_{1}^{\prime}=E_{c}^{\prime}=0$. We find $\left|E_{\mathrm{S}}\right|=$ $\left(\gamma_{m} \tau_{r} T_{\mathrm{S}} / \gamma_{\mathrm{abs}}\right)^{1 / 2}$ and

$$
P_{\text {in }}=\frac{4 \pi^{2} \gamma_{m} \tau_{r}}{\kappa^{2} \gamma_{\text {abs }}} T_{\mathrm{S}}\left\{\left[v_{1}(1+\chi) T_{\mathrm{S}}+\Delta\right]^{2}+Y^{2}\right\},
$$

where we have defined

$$
\begin{gathered}
\chi=\gamma_{m} N_{k} /\left(\nu_{1} \gamma_{\mathrm{abs}}\right)=\gamma_{m} n_{k} /\left(A \beta \gamma_{\mathrm{abs}}\right), \\
Y=\xi_{0}^{2} \chi /\left(\pi \gamma_{m}\right)=-q /(2 \pi), \\
\xi_{0}^{2}=-q \nu_{1} \gamma_{\mathrm{abs}} /\left(2 N_{k}\right)=-q A \beta \gamma_{\mathrm{abs}} /\left(2 n_{k}\right) .
\end{gathered}
$$

Here, $\chi$ is dimensionless and $\xi_{0}$ is a characteristic frequency. Note that $\chi<0$. Equation (8) implicitly defines the stationary temperatures for a given input power. The right-hand side of Eq. (8), plotted in Fig. 4, is cubic in $T_{\mathrm{S}}$, so Eq. (8) can have one, two, or three real solutions, depending on the left-hand side. The existence of two stationary solutions corresponds to a bistability and occurs when the high-temperature minimum of the right-hand side exactly equals the left-hand side. This condition is indicated by the dashed, orange lines in Fig. 4. The high-temperature minimum occurs at a critical temperature:

$$
\begin{aligned}
T_{\mathrm{C}} & =-\frac{2 \Delta+\sqrt{\Delta^{2}-3 Y^{2}}}{3(1+\chi) v_{1}}, \\
& =T_{0}\left[1-Y^{2} /\left(2 \Delta^{2}\right)+O(Y / \Delta)^{4}\right],
\end{aligned}
$$


TABLE I. Parameter values used in simulations. The first block of parameters lists physical and geometrical quantities, the next block lists calculated quantities that appear explicitly in the model, and the final block lists reduced parameters that determine the dynamics. (Note that both $T_{0}$ and $P_{0}$ depend on $\Delta$, and the values above assume a typical value of $\Delta=4 \mathrm{MHz} \gtrsim \Delta_{0}$, for didactic purposes.)

\begin{tabular}{lc}
\hline \hline Parameter & Value \\
\hline$r$ & $5 \mathrm{~mm}$ \\
$A$ & $1.2 \times 10^{-9} \mathrm{~m}^{2}$ \\
$n$ & 1.43 \\
$f_{r}$ & $281.9 \mathrm{THz}$ \\
$\beta$ & $-1.14 \times 10^{-5} / K$ \\
$n_{k}$ & $1.9 \times 10^{-20} \mathrm{~m}^{2} / \mathrm{W}$ \\
$Q_{0}$ & $7 \times 10^{8}$ \\
$Q_{c}$ & $8 \times 10^{8}$ \\
$q^{2}$ & $2.37 \mathrm{MHz}$ \\
$\kappa^{2}$ & $2.21 \mathrm{MHz}$ \\
$\gamma_{m}$ & $2750 / \mathrm{s}$ \\
$\gamma_{\mathrm{abs}}$ & $45.0 \mathrm{mK} / \mathrm{J}$ \\
$\tau_{r}$ & $150 \mathrm{ps}$ \\
$\nu_{1}$ & $-2.25 \mathrm{GHz} / \mathrm{K}$ \\
$N_{k}$ & $3.12 \mathrm{kHz} / \mathrm{W}$ \\
\hline$\chi$ & -0.085 \\
$Y$ & $-378 \mathrm{kHz}$ \\
$\xi_{0}$ & $196 \mathrm{kRad} / \mathrm{s}$ \\
$T_{0}$ & $1.94 \mathrm{mK}$ \\
$P_{0}$ & $45.1 \mathrm{~mW}$ \\
$\Delta_{0}$ & $3.67 \mathrm{MHz}$ \\
\hline \hline
\end{tabular}

where $T_{0}=-\frac{\Delta}{(1+\chi) \nu_{1}}>0$. The corresponding critical power $P_{\mathrm{C}}$ is the value implied by Eq. (8) at $T_{\mathrm{S}}=T_{\mathrm{C}}$ and is given by

$$
P_{\mathrm{C}}=P_{0}\left[1-Y^{2} /\left(4 \Delta^{2}\right)+O(Y / \Delta)^{4}\right],
$$

where $P_{0}=\frac{4 \pi^{2} \gamma_{m} \tau_{r}}{\kappa^{2} \gamma_{\text {abs }}} T_{0} Y^{2}$.

We note from Eq. (12) that there is no high-temperature stationary solution if $|\Delta|<\left|\Delta_{\mathrm{C}}^{\min }\right|=\sqrt{3}|Y|$. The onset of

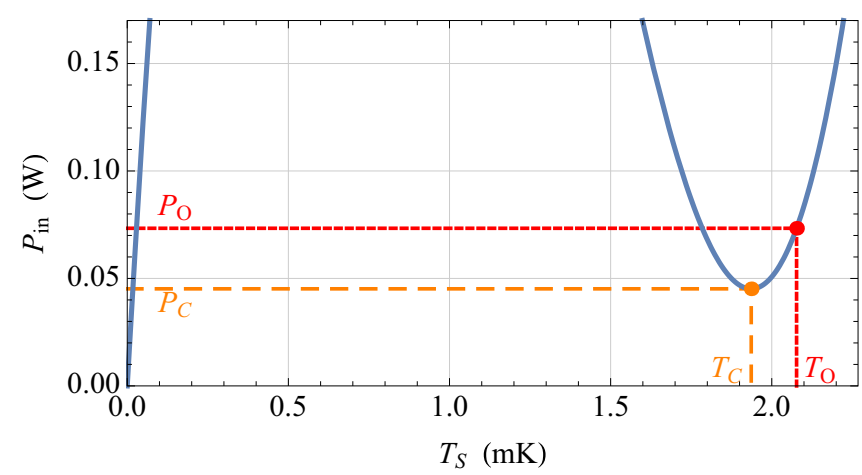

FIG. 4. (Color online) The solid blue lines are the cubic expression on the right-hand side of Eq. (8). The dashed orange lines indicate the critical temperature and power above which a high-temperature stationary solution exists. The dash-dotted red lines indicate the temperature and power above which self-sustained oscillations emerge. Numerical parameter values are as in Table I, and we assume $\Delta=4 \mathrm{MHz}>\Delta_{0}$. the high-temperature solution at $\left|\Delta_{\mathrm{C}}^{\min }\right|$ occurs at $T_{\mathrm{C}}^{\mathrm{min}}=$ $-\frac{2|Y|}{\sqrt{3}(1+\chi) v_{1}}=(2 / 3) T_{0}^{\mathrm{min}}$ and $P_{\mathrm{C}}^{\mathrm{min}}=(8 / 9) P_{0}^{\min }$.

For powers just above $P_{\mathrm{C}}$, the high-temperature stationary solution is stable along the right-hand branch in Fig. 4. At sufficiently high power, the high-temperature solution exhibits a Hopf instability, where the stable, high-temperature stationary state is replaced by a limit cycle characterized by self-sustaining oscillations. The criterion for the Hopf instability is that the real part of a pair of eigenvalues of the Jacobian [31] associated with the dynamical system changes sign from negative (stable) to positive (unstable) [32]. The characteristic equation for the Jacobian is

$$
0=-\lambda^{3}+b \lambda^{2}+c \lambda+d
$$

where

$$
\begin{aligned}
& b=4 \pi Y-\gamma_{m}, \\
& c=4 \pi^{2}\left\{\left(v_{1} T_{1} \chi\right)^{2}-\left[\Delta+(1+2 \chi) \nu_{1} T_{1}\right]^{2}-Y\left(Y-\gamma_{m} / \pi\right)\right\}, \\
& d=4 \pi^{2} \gamma_{m}\left\{\Delta^{2}-\left[2 \Delta+3(1+\chi) \nu_{1} T_{1}\right]^{2}-3 Y^{2}\right\} / 3 .
\end{aligned}
$$

When the real part of a pair of mutually conjugate eigenvalues vanishes, they become pure imaginary: $\lambda_{ \pm}= \pm i \xi$. At this point, the characteristic equation becomes

$$
0= \pm i \xi\left(\xi^{2}+c\right)-b\left(\xi^{2}-d / b\right) .
$$

Solving the real and imaginary parts of Eq. (15) for $\xi$ gives two separate conditions: $-c=\xi^{2}=d / b$. It follows that $c+$ $d / b=0$, which we then solve for $T_{1}$ to find the temperature, $T_{\mathrm{O}}$, power, $P_{\mathrm{O}}$, and characteristic frequency, $\xi$, at the onset of the oscillations arising from the Hopf instability. We find that $T_{\mathrm{O}}>T_{\mathrm{C}}$ and $P_{\mathrm{O}}>P_{\mathrm{C}}$, as indicated by the red, dotted lines in Fig. 4. Figure 5(a) shows the characteristic frequency, $\xi$, as a function of detuning. Figure 5(b) shows the power, $P_{\mathrm{O}}$, at which oscillations become self-sustaining, as a function of detuning.

The analytic expressions for these quantities are somewhat cumbersome, but asymptotic expansions for large $\Delta$ are as follows:

$$
\begin{aligned}
& T_{\mathrm{O}}=T_{0}\left(1-\frac{(1+\chi) Y^{2}}{2 \chi \Delta^{2}}+\frac{(1+\chi)(1+4 \chi) \gamma_{m} Y}{8 \pi \chi^{2} \Delta^{2}}+\cdots\right), \\
& P_{\mathrm{O}}=P_{0}\left(1+\frac{\left(1-\chi^{2}\right) Y^{2}}{4 \chi^{2} \Delta^{2}}-\frac{(1+\chi)(1+4 \chi) \gamma_{m} Y}{8 \pi \chi^{3} \Delta^{2}}+\cdots\right), \\
& \xi^{2}=\xi_{0}^{2}\left(1+\frac{(1+\chi)^{2} Y^{2}}{4 \chi^{2} \Delta^{2}}-\frac{(1+\chi)^{2}(3+8 \chi) \gamma_{m} Y}{16 \pi \chi^{3} \Delta^{2}}+\cdots\right),
\end{aligned}
$$

where "..." includes the terms $O\left(\frac{Y^{4}}{\Delta^{4}}, \frac{\gamma_{m}^{2}}{\Delta^{2}}\right)$.

We note that $c+d / b=0$ has no real solution if $|\Delta|<$ $\Delta_{\mathrm{O}}^{\min }$, where

$$
\begin{aligned}
\Delta_{\mathrm{O}}^{\min } & =\frac{\left(\gamma_{m}-2 \pi Y\right) \sqrt{2(1+\chi)\left[\gamma_{m} / \pi+2(1+3 \chi) Y\right] Y}}{\gamma_{m}+4 \pi \chi Y} \\
& =\Delta_{0}\left(1-\frac{\left(1+4 \chi+6 \chi^{2}\right) \gamma_{m}}{4 \pi \chi(1+3 \chi) Y}+O\left(\gamma_{m} / Y\right)^{2}\right),
\end{aligned}
$$

where $\Delta_{0}=\sqrt{(1+\chi)(1+3 \chi)} Y / \chi$. That is, for sufficiently small detuning, there may be no Hopf instability. This minimum detuning is shown as a dotted vertical line in Fig. 5. 

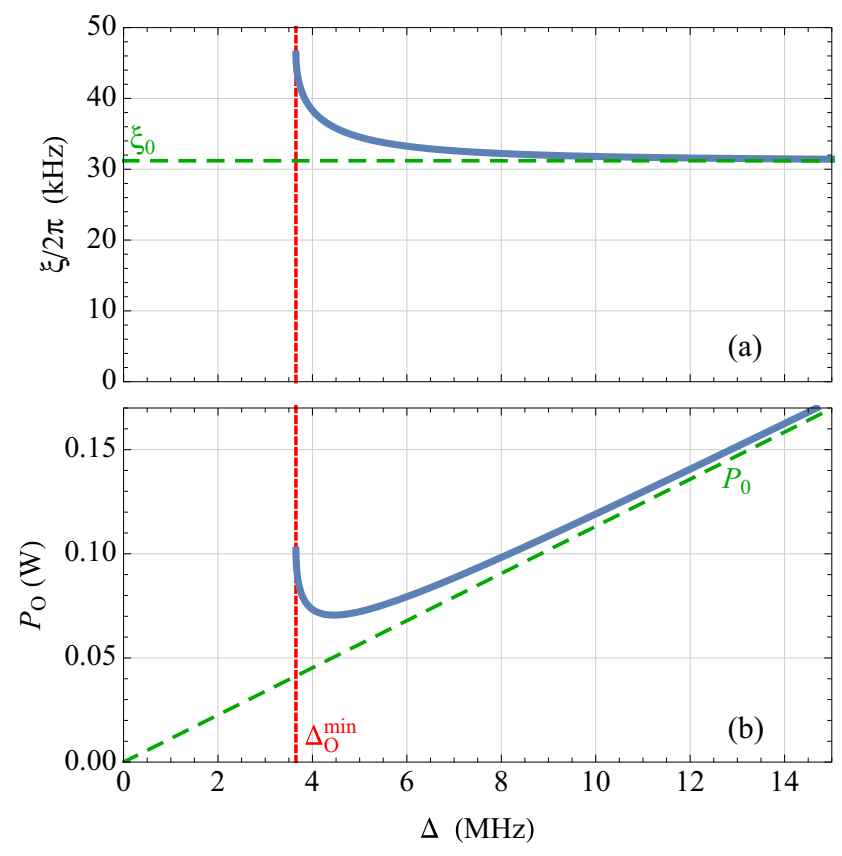

(a)

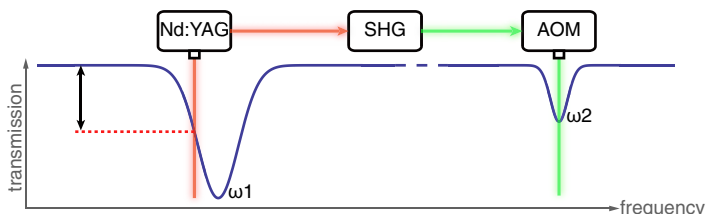

(b)

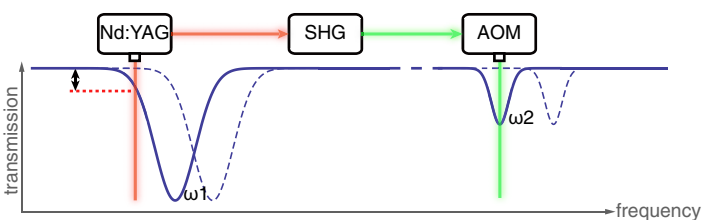

(c)

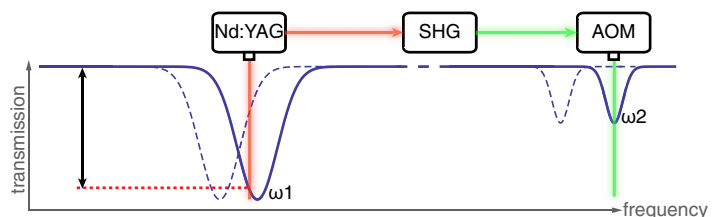

FIG. 5. (Color online) (a) Oscillation frequency, $\xi$ (solid, blue line), versus detuning; asymptotic frequency, $\xi_{0} / 2 \pi$ (dashed, green line). (b) Critical power, $P_{\mathrm{O}}$ (solid, blue line), for onset of oscillation versus detuning; asymptotic power, $P_{0}$ (dashed, green line). Numerical parameter values are as in Table I.

Using our theoretical model, we calculate the limit-cycle oscillation frequency, $\xi \sim 34-50 \mathrm{kHz}$ [depending on detuning, see Fig. 5(a)], which is consistent with the experimental results and simulations in Figs. 3(i), 3(j), and 3(1). The existence of the Hopf instability means that the presence or absence of oscillatory behavior in, e.g,. Figures 3(i) and 3(1) is sensitive to the pump power, detuning, and material properties. We calculate the critical power for the onset of oscillations, $P_{\mathrm{O}} \gtrsim 70 \mathrm{~mW}$ [see Fig. 5(b)], which is marginally smaller than, and consistent with, the experimental condition in Figs. 3(i) and 3(1).

\section{DUAL-MODE SELF-THERMAL- LOCKING TECHNIQUE}

To suppress the oscillatory behavior at strong driving power, we develop a dual-mode self-thermal-locking technique by combining a thermal self-locking technique [24] with a dualmode temperature-sensing technique [33]. This combination not only locks the resonator frequency to the laser frequency (as happens in the traditional technique) but additionally has the effect of automatically stabilizing the temperature of the resonator.

The principles of the approach are illustrated in Fig. 6: a weak frequency-doubled signal (532 nm in this work) is frequency shifted by an acousto-optic modulator (AOM) and locked to a resonator mode at a frequency of $\omega_{2}$ with an active locking technique (e.g., the Pound-Drever-Hall (PDH) technique [34]); i.e., the control system has achieved $2 \omega_{l}+$ $\omega_{\mathrm{AOM}}=\omega_{2}$, where $\omega_{l}$ is the laser fundamental frequency and $\omega_{\mathrm{AOM}}$ is the frequency of the up-shifting AOM. Then a much

FIG. 6. (Color online) (a) The frequency-shifted second harmonic (green output of "SHG") is locked to a WGM resonance so that the 1064-nm Nd:YAG laser output sits on the red-detuned side of a low-frequency mode. (b) When the mode temperature increases, the 1064-nm laser shifts further from resonance. This decreases the in-coupled power (proportional to the height of the vertical arrows), thereby decreasing the optical heating rate. (c) When the mode temperature decreases, the 1064-nm laser shifts closer to resonance. This increases the in-coupled power, thereby increasing the optical heating rate. SHG, second-harmonic generation. The dashed curves in panels (b) and (c) are for reference only.

stronger laser signal at the fundamental wavelength $(1064 \mathrm{~nm}$ in this work) is detuned, $\Delta \omega_{1}=\omega_{l}-\omega_{1}=\left(\omega_{2} / 2-\omega_{1}\right)-$ $\omega_{\mathrm{AOM}} / 2$, with respect to a second mode at a frequency of $\omega_{1}$ in the same resonator by tuning the AOM frequency to an appropriate value. Ideally the two resonator modes at different wavelengths should belong to the same mode family to achieve maximum spatial overlap. The frequency difference between these two modes, $\omega_{2} / 2-\omega_{1}$, is an excellent proxy for the temperature of the resonator averaged over the mode volume while it is nearly independent of the temperature elsewhere in the resonator [33]. If the resonator temperature decreases then the detuning between the 1064-nm laser frequency and the resonant mode will decrease, leading to an increased coupledin power. The additional heat generated by the decreased detuning will lead to a compensatory increase in the resonator temperature. On the other hand, a rise in temperature will reduce the heating by the $1064-\mathrm{nm}$ beam. We see a natural equilibrium position in which the fundamental laser signal will be locked to the red side of the mode while any environmental temperature fluctuations can be suppressed. Furthermore, the insensitivity to thermal expansion within the resonator means that the conditions required for unwanted thermal oscillations will also be overcome.

The self-locking technique makes use of the experimental arrangement illustrated in Fig. 7. We combine $20 \mu \mathrm{W}$ of the 532-nm second-harmonic generation output of the Nd:YAG laser with about $80 \mathrm{~mW}$ of the 1064-nm output light and couple both beams into the resonator. The 532-nm beam passes through an AOM for frequency tuning so it can be 


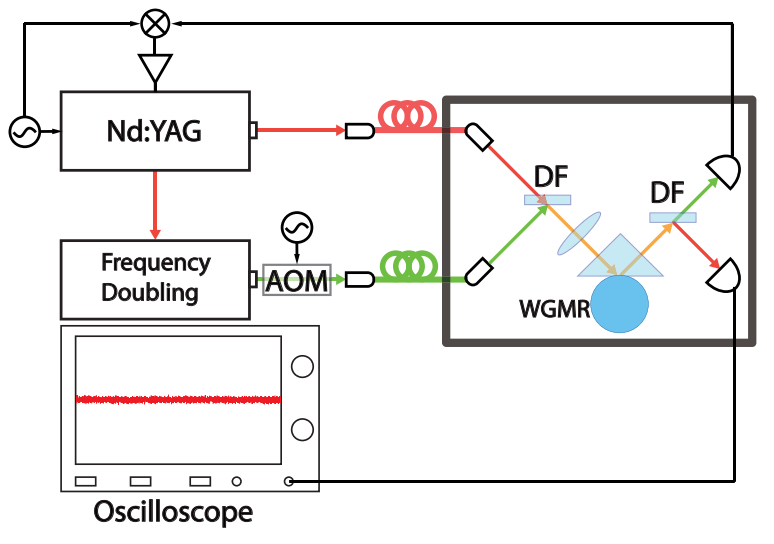

FIG. 7. (Color online) Experimental scheme for implementing the dual-mode self-thermal-locking technique. The oscilloscope was for monitoring the fundamental signal coupling depth. DF, dichroic filter.

brought onto resonance. The transmitted light is separated by another dichroic mirror and registered by two photodetectors. The 532-nm signal is actively frequency locked to a mode with the PDH technique by feedback to the generated error signal into the frequency modulation port of the laser. The fundamental signal of $1064 \mathrm{~nm}$ is set on the red-detuned side (at a frequency corresponding to approximately half the maximum throughput) of a second mode within the same mode family - this is achieved by adjustment of the AOM on the 532-nm beam. This arrangement allows us to maintain resonance for many hours with a highly stable temperature. We use a frequency counter to measure the difference frequency between the 1064-nm signal and that of a single mode in a highly stabilized frequency comb $\left(\Delta f / f \sim 10^{-14}\right)$.

Figure 8 (a) shows the resulting stabilized output frequency over 5 h, while Fig. 8(b) demonstrates the benefit of the self-locking scheme in terms of the frequency instability. One can see that this technique not only allows coupling of high power into the resonator at its red-detuned side without causing thermal oscillations but also suppresses modefrequency fluctuations.

It is important to note that the entire resonator temperature is not stabilized by this technique but only the temperature within the optical mode volume. Any environmental changes will still induce temperature gradients and consequently cause a mode resonance frequency shift. Nonetheless, the resonator frequency was stabilized to better than $2 \mathrm{MHz}$ as seen over $5 \mathrm{~h}$ in Fig. 8(a). Further substantial improvement could be achieved with some modest passive temperature shielding of the resonator.

Here we analyze the optimal operational condition of this technique. Using the results from Eqs. (1)-(4) and the analysis developed in Ref. [33] the thermal-locked mode temperature evolves as

$$
\frac{d T_{m}}{d t}=\gamma_{\mathrm{abs}} \eta P_{\mathrm{in}}-\gamma_{m}\left(T_{m}-T_{r}\right),
$$

where $\eta=\frac{\omega_{0}}{\tau_{r} Q_{c}} \frac{1}{(\delta / 2)^{2}+\Delta \omega_{1}^{2}}$ is proportional to the power build-up factor, while $\Delta \omega_{1}$ can be expressed in terms of $T_{m}$ and $P_{\text {in }}$ as

$$
\Delta \omega_{1}=C+2 \omega_{1} \beta_{\mathrm{eff}} T_{m}+2 \omega_{1} n_{\mathrm{k}} \eta A_{\mathrm{eff}}^{-1} P_{\mathrm{in}},
$$
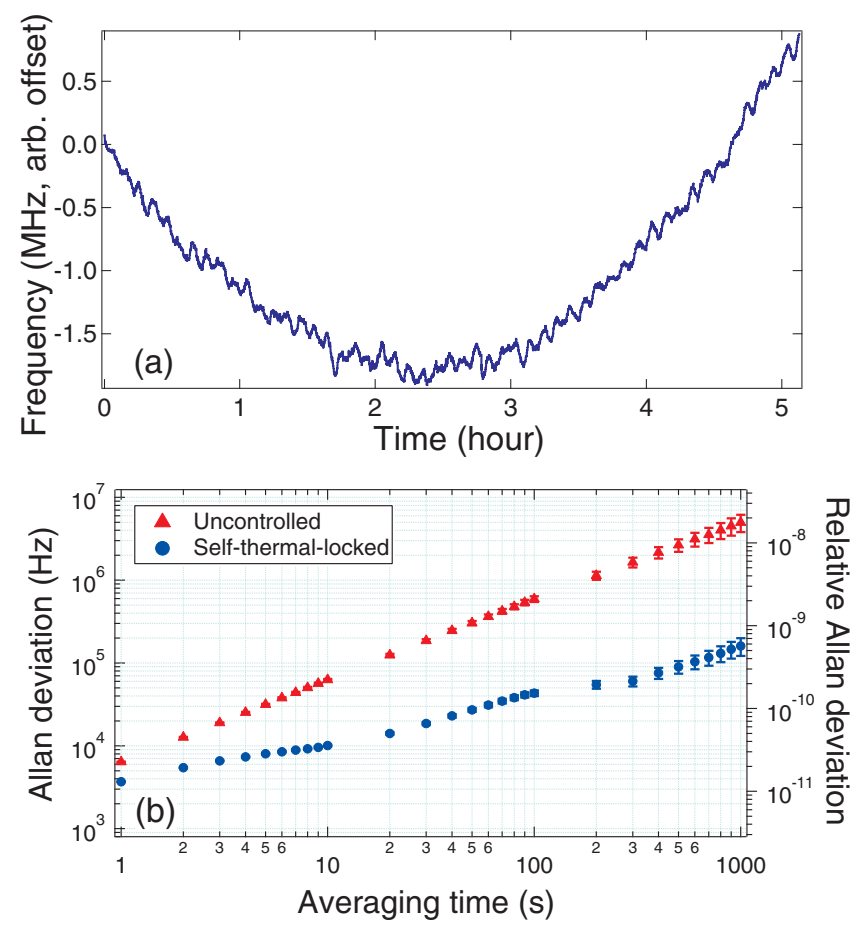

FIG. 8. (Color online) (a) The 1064-nm frequency measured with a frequency comb when the dual-mode self-thermal-locking technique was implemented. (b) The fundamental frequency Allan deviations when the self-thermal-locking technique was implemented (blue circles) and when it was not (red triangles).

where $C$ is a constant, $\beta_{\text {eff }}=\frac{\beta_{1}}{n_{1}}-\frac{\beta_{2}}{n_{2}}$, and $\beta_{1}, \beta_{2}, n_{1}$, and $n_{2}$ are the thermooptic coefficients and refractive indices at the wavelengths of the fundamental signal and the second harmonic, respectively, while the effective mode overlap is given by $A_{\text {eff }}^{-1}=\left(n_{1} A_{1}\right)^{-1}-\left(n_{2} A_{2}\right)^{-1}$, where $A_{1}$ and $A_{2}$ are the effective cross-sectional areas of the modes [33].

Following the stabilization analysis in Refs. [24,35] we can derive some important results for optimal operation of this autostabilization. By considering operation when the mode temperature is stabilized $\left(\frac{d T_{m}}{d t}=0\right)$, we can derive from Eq. (17) that the mode temperature dependence on the resonator temperature is

$$
\frac{d T_{m}}{d T_{r}}=\frac{1}{1+\frac{\gamma_{\mathrm{abs}}}{\gamma_{m}} \frac{\zeta \beta_{\mathrm{eff}} \Delta \omega_{1}}{\left[\left(\frac{\delta}{2}\right)^{2}+\Delta \omega_{1}^{2}\right]^{2}+\zeta n_{\mathrm{k}} A_{\mathrm{eff}}^{-1} \Delta \omega_{1}}},
$$

where $\zeta=\frac{4 \omega_{1}^{2}}{\tau_{r} Q_{c}} P_{\text {in }}$. One notes that there is a minimum in this expression when $\Delta \omega_{1}=-\frac{\delta}{2 \sqrt{3}}$, which thus sets the optimal operational point. This means that at this frequency detuning the mode volume temperature will be least affected by temperature changes in the rest of the resonator and hence the mode frequency will be most stable. Similarly, we find that the dependence of the mode temperature on the input power is

$$
\frac{d T_{m}}{d P_{\mathrm{in}}}=\frac{\gamma_{\mathrm{abs}}}{\gamma_{m}} \eta\left(1-4 \eta^{2} \tau_{r} Q_{c} \Delta \omega_{1} n_{\mathrm{k}} A_{\mathrm{eff}}^{-1} P_{\mathrm{in}}\right) .
$$


We see in this case that when $P_{\text {in }}=\frac{A_{\text {eff }}}{4 \eta^{2} \tau_{r} Q_{c} \Delta \omega_{1} n_{\mathrm{k}}}$ the dependence will be eliminated $\left(d T_{m} / d P_{\text {in }}=0\right)$ and hence input power fluctuations will not result in temperature fluctuations.

\section{CONCLUSION}

In conclusion, dynamical thermal and mode-frequency oscillations at different time scales were explored both experimentally and theoretically. A self-referencing locking technique was proposed and demonstrated. We show that this technique can couple high-intensity power into a $\mathrm{CaF}_{2}$ resonator and automatically stabilize both the temperature and the frequency. With a simple modification, this technique could also be used in anisotropic resonators by using excitation in two different polarizations [36,37]. Alternatively, one can envisage self-generation of the two required signals from a single input signal using parametric processes (such as occurs in comb generation or parametric amplification).

\section{ACKNOWLEDGMENTS}

The authors acknowledge support from the Australian Research Council under Grants No. DP0877938, No. FT0991631, No. LE110100054, and No. LE100100009. The authors also wish to acknowledge the South Australian Government who have provided generous financial support through the Premier's Science and Research Fund. The authors thank Geoff Campbell for shaping the resonator and Ping Koy Lam for developing the diamond turning facility. W.W. gratefully acknowledges financial support from the China Scholarship Council and the University of Western Australia.
[1] K. J. Vahala, Nature (London) 424, 839 (2003).

[2] T. J. Kippenberg and K. J. Vahala, Opt. Express 15, 17172 (2007).

[3] T. J. Kippenberg, A. L. Tchebotareva, J. Kalkman, A. Polman, and K. J. Vahala, Phys. Rev. Lett. 103, 027406 (2009).

[4] D. J. Alton, N. P. Stern, T. Aoki, H. Lee, E. Ostby, K. J. Vahala, and H. J. Kimble, Nat. Phys. 7, 159 (2011).

[5] F. Vollmer, S. Arnold, and D. Keng, Proc. Natl. Acad. Sci. USA 105, 20701 (2008).

[6] J. Zhu, S. K. Ozdemir, Y.-F. Xiao, L. Li, L. He, D.-R. Chen, and L. Yang, Nat. Photonics 4, 46 (2009).

[7] T. Kippenberg, R. Holzwarth, and S. Diddams, Science 332, 555 (2011).

[8] G. Bahl, K. H. Kim, W. Lee, J. Liu, X. Fan, and T. Carmon, Nat. Commun. 4, 1994 (2013).

[9] I. S. Grudinin, V. S. Ilchenko, and L. Maleki, Phys. Rev. A 74, 063806 (2006).

[10] B. Sprenger, H. Schwefel, Z. Lu, S. Svitlov, and L. Wang, Opt. Lett. 35, 2870 (2010).

[11] I. S. Grudinin, N. Yu, and L. Maleki, Opt. Lett. 34, 878 (2009).

[12] A. A. Savchenkov, A. B. Matsko, M. Mohageg, and L. Maleki, Opt. Lett. 32, 497 (2007).

[13] A. Savchenkov, A. Matsko, V. Ilchenko, D. Seidel, and L. Maleki, Opt. Lett. 36, 3338 (2011).

[14] J. Hofer, A. Schliesser, and T. J. Kippenberg, Phys. Rev. A 82, 031804 (2010).

[15] L. He, Y.-F. Xiao, J. Zhu, S. K. Ozdemir, and L. Yang, Opt. Express 17, 9571 (2009).

[16] A. E. Fomin, M. L. Gorodetsky, I. S. Grudinin, and V. S. Ilchenko, J. Opt. Soc. Am. B 22, 459 (2005).

[17] Y.-S. Park and H. Wang, Opt. Lett. 32, 3104 (2007).

[18] Y. Deng, R. Flores-Flores, R. K. Jain, and M. Hossein-Zadeh, Opt. Lett. 38, 4413 (2013).

[19] M. Soltani, S. Yegnanarayanan, Q. Li, A. A. Eftekhar, and A. Adibi, Phys. Rev. A 85, 053819 (2012).

[20] L. Zhang, Y. Fei, T. Cao, Y. Cao, Q. Xu, and S. Chen, Phys. Rev. A 87, 053805 (2013).
[21] M. L. Gorodetsky and V. S. Ilchenko, J. Opt. Soc. Am. B 16, 147 (1999).

[22] P. Del'Haye, T. Herr, E. Gavartin, M. L. Gorodetsky, R. Holzwarth, and T. J. Kippenberg, Phys. Rev. Lett. 107, 063901 (2011)

[23] C. Wang, T. Herr, P. Del'Haye, A. Schliesser, J. Hofer, R. Holzwarth, T. Hänsch, N. Picqué, and T. Kippenberg, Nat. Commun. 4, 1345 (2013).

[24] T. Carmon, L. Yang, and K. Vahala, Opt. Express 12, 4742 (2004).

[25] Data from Corning, techical report, https://lightmachinery.com/ media/1542/h0607_caf2_product_sheet.pdf.

[26] V. Ilchenko and M. Gorodetskii, Laser Phys. 2, 1004 (1992).

[27] B. Fan, Mesoscopic Quantum Sensors, Ph.D. thesis, University of Queensland, 2014.

[28] T. Carmon, H. Rokhsari, L. Yang, T. J. Kippenberg, and K. J. Vahala, Phys. Rev. Lett. 94, 223902 (2005).

[29] F. Marquardt, J. G. E. Harris, and S. M. Girvin, Phys. Rev. Lett. 96, 103901 (2006).

[30] D. A. Rodrigues and A. D. Armour, Phys. Rev. Lett. 104, 053601 (2010).

[31] The Jacobian is defined by introducing an additional dynamical equation for $E_{c}^{*}$ and treating $T_{1}, E_{c}$, and $E_{c}^{*}$ as formally independent variables, leading to a cubic characteristic equation.

[32] S. H. Strogatz, Nonlinear Dynamics and Chaos: With Applications to Physics, Biology and Chemistry (Perseus, New York, 2001).

[33] W. Weng, J. D. Anstie, T. M. Stace, G. Campbell, F. N. Baynes, and A. N. Luiten, Phys. Rev. Lett. 112, 160801 (2014).

[34] R. Drever, J. L. Hall, F. Kowalski, J. Hough, G. Ford, A. Munley, and H. Ward, Appl. Phys. B 31, 97 (1983).

[35] I. Teraoka, Opt. Commun. 310, 212 (2014).

[36] D. V. Strekalov, R. J. Thompson, L. M. Baumgartel, I. S. Grudinin, and N. Yu, Opt. Express 19, 14495 (2011).

[37] I. Fescenko, J. Alnis, A. Schliesser, C. Wang, T. Kippenberg, and T. Hänsch, Opt. Express 20, 19185 (2012). 